\title{
The Clinical Impact of the Late Imaging with 18F-Fluorodeoxyglucose PET Texture Analysis in Invasive Lobular Breast Cancer
}

\author{
(D) Fikri Okan FALAY, (1) Hülya SEYMEN
}

Department of Nuclear Medicine, Molecular Imaging and Treatment, Koç University Faculty of Medicine, Istanbul-Turkey

\begin{abstract}
OBJECTIVE
Breast invasive lobular cancer (ILC) is one of the most difficult malignancies to diagnose and follow-up due to its tumor morphology. Tumor heterogeneity is the most important reason for treatment failure and diagnostic limitation. Identification of heterogeneity by a non-invasive method, texture analysis that can be done from positron emission tomography (PET), MR, and CT is developed. In ILC, diagnostic sensitivity is lower with $18 \mathrm{~F}$-fluorodeoxyglucose ([18F] FDG) PET/CT compared to invasive ductal carcinoma (IDC). In this study, the correlation between histopathological variables and the texture analysis of [18F] FDG PET/CT standard images, and also late images whose contribution to the diagnosis of many malignancies has been investigated are researched.
\end{abstract}

\section{METHODS}

Twenty ILC patients underwent standard and late [18F] FDG PET/CT imaging for staging between May 2007 and December 2018. T and N stages, histological and nuclear grades, estrogen receptor, progesterone receptor, human epidermal growth factor-2, and Ki-67 were recorded.

\section{RESULTS}

Thirty-two textural indices with conventional and formal indices and histogram values were calculated with LIFEx software to find heterogeneity in standard and late [18F] FDG PET/CT images. Conventional and discrete indices based on GLRM and GLZM are more correlated than other texture indices. Greater number of significantly correlations was found between histopathological variables and texture analysis of late imaging $(\mathrm{p}<0.05)$.

\section{CONCLUSION}

In our study, the conventional indices, especially in the $2^{\text {nd }}$ degree indices, in the texture analysis performed with [18F] FDG PET/CT significantly correlated in the ILC, which has lower [18F] FDG affinity compared to IDC due to the tumoral tissue characteristics. Although the presence of more correlations with histopathological prognostic information in late images suggests a greater diagnostic contribution, further studies with more numbers are needed.

Keywords: [18F] FDG PET/CT; breast cancer; late imaging; texture analysis.

Copyright $\odot$ 2021, Turkish Society for Radiation Oncology

\section{Introduction}

According to the 2017 data of the American Cancer Society, breast cancer is the most common cancer in women.
Breast cancer ranks second in cancer-related deaths after lung cancer.[1] Early diagnosis, more accurate and non-intervention staging, follow-up of treatment, and prognosis are the most important processes in deter- 
mining the approach to breast cancer.[2] About 50-70\% of breast cancers are invasive ductal carcinoma (IDC), $5-15 \%$ are invasive lobular cancer (ILC), 1-6\% are mucinous carcinoma, and 1-2\% are tubular carcinoma.[3] The diagnosis and staging of ILC is more difficult than IDC because of the characteristic growth pattern.[4] The tumor does not produce a stromal reaction and therefore tissue thickening occurs more than a mass formation and microcalcification, which is the most important clinical finding in early diagnosis that is rarely seen. $[5,6]$

Although 18F-fluorodeoxyglucose ([18F] FDG) positron emission tomography (PET) imaging has high sensitivity and specificity in showing malignant lesions, its sensitivity in breast cancer is limited. In a meta-analysis, the overall sensitivity and specificity of [18F] FDG PET/CT in detecting primary breast cancer were found to be $64-96 \%$ and $73-100 \%$, respectively.[7] While the sensitivity of [18F]FDG PET/CT is $57 \%$ in tumors $<1 \mathrm{~cm}$ in diameter, it exceeds $90 \%$ in tumors larger than $1 \mathrm{~cm}$.[8] Glut-1 and hexokinase expression, number of viable tumor cells, histological subgroup, microvascular density, and presence of inflammatory cells are the major factors affecting [18F] FDG uptake in breast cancer.[9] It is known that estrogen and progesterone receptor negative, human epidermal growth factor receptor 2 (HER-2) expression positive tumors show higher [18F] FDG uptake than receptor-positive and HER-2-negative tumors.[10] Furthermore, [18F] FDG PET/CT imaging is less sensitive in ILC than IDC because of low [18F] FDG uptake. It is reported that the clinical systemic contribution of PET/CT is low due to the low [18F] FDG affinity in mucinous (1-6\%) and tubular (1-2\%) cancers, which are less common with ILC, which constitutes $10-15 \%$ of breast cancers. $[11,12]$ The main factors that explain low [18F] FDG uptake in ILCs are lower tumor cell density, diffuse infiltration in the surrounding tissue, low Glut-1 expression, and low proliferation rate.[13] For these reasons, the influence of [18F] FDG PET/CT to IDC was mostly assessed in the studies and the ILC could be included in a limited number of studies or was not included because of low sensitivity. $[8,14]$

It is known that the continued accumulation of [18F] FDG is due to the low concentration of glucose-6-phosphatase enzyme in the tumor cell, and it is known that imaging quality will be optimal as long as the radiopharmaceutical half-life allows and that imaging may contribute to the diagnosis in malignancies. [15] There are many studies reporting that late image on [18F] FDG PET/CT significantly increases sensitivity and specificity in breast cancer.[16-18]
Last decade, the importance of measuring tumor heterogeneity is identified to solve the inability of current therapies. The tumor heterogeneity is not fully understood with invasive techniques, such as biopsy, and also non-invasive imaging techniques such as PET/CT which could give the information of genotypic and phenotypic tumor variations. Many techniques have been proposed to use tumor heterogeneity as a biomarker. There are some mathematical methods to define tumor heterogeneity. One of these is the term "texture analysis." This analysis specifies the various quantification of the spatial distribution of voxel intensities in images. The advantages of using imaging techniques to define heterogeneity are the account of the whole tumor, intratumoral heterogeneity in very small spatial scales, and non-invasiveness.[19] However, in daily practice, these methods are barely used.

In this retrospective study, we aimed to investigate the effect of late imaging and textural analysis on diagnosis in pre-operative [18F] FDG PET/CT of ILC.

\section{Materials and Methods}

\section{Patients}

Between May 2007 and December 2018, 20 patients referred for [18F] FDG PET/CT for the staging of an invasive lobular breast cancer to the department of nuclear medicine and molecular imaging were included in this study.

\section{Image Acquisition}

About 50-70 min after intravenous 5-9 mCi [18F]FDG, standard 7-9 bed length between vertex and mid-crus and after 160-200 min, neck and thorax 2-bed area late imaging were taken Discovery IQ 5 ring (General Electric Corp., Milwaukee, WI, USA) PET/CT device.

\section{Images Analysis}

All primary tumors of standard and late [18F] FDG PET/CT images were delineated with $40 \%$ of SUV maximum value method to create volumes of interest (VOI). The texture analysis results were recorded with s-prefix for standard images and l-prefix for late images. In each VOI, standard PET quantitative measures were evaluated by conventional indices (SUVmax, SUVmin, SUVmean, SUVstd, SUVpeak, and TLG), indices from histogram (skewness and kurtosis) and shape (MTV, sphericity, and capacity) and 32 textural indices were recorded using LIFEx software (Fig. 1). The subtraction of these textural indicis was created by calculating four different matrices for each 


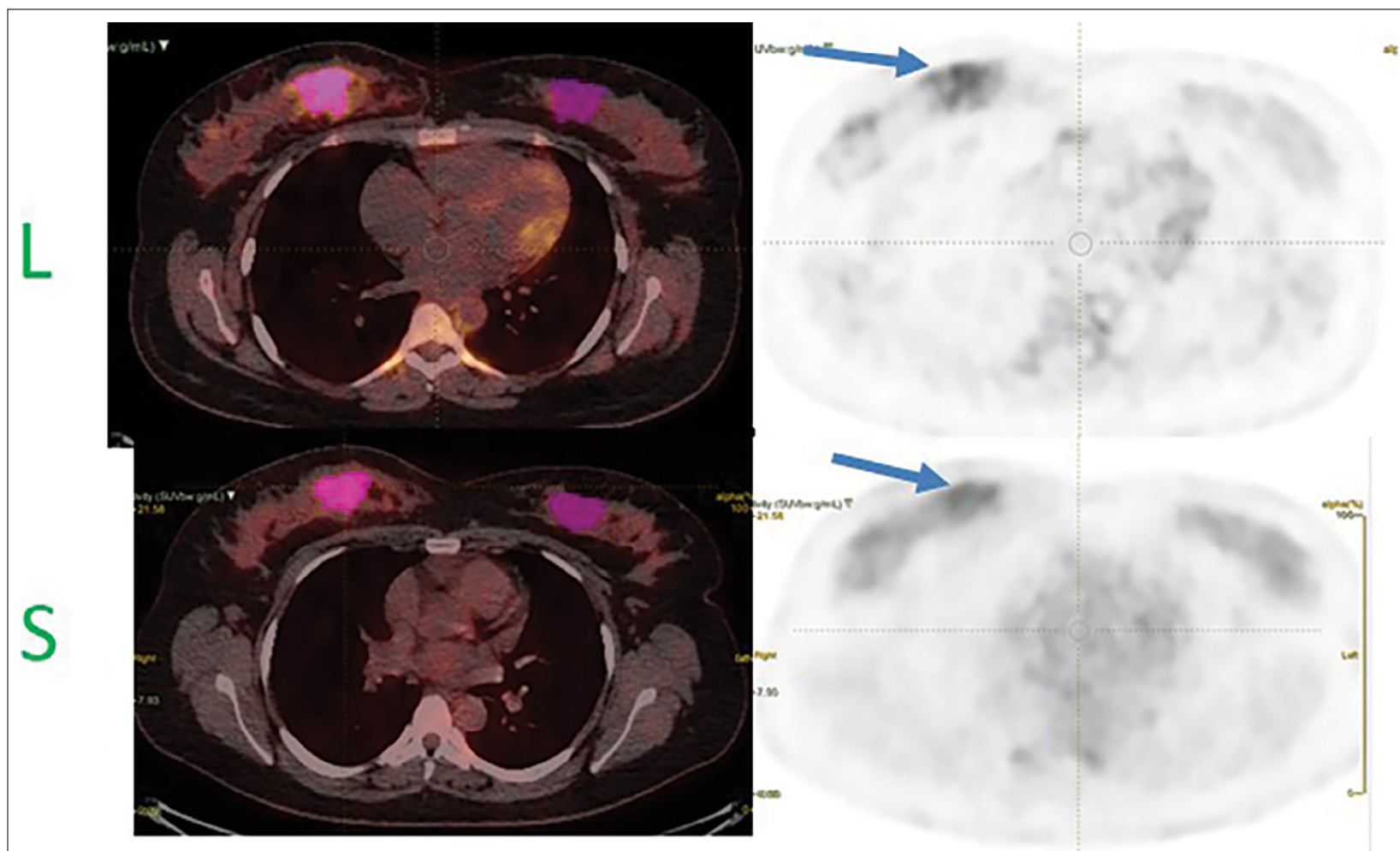

Fig. 1. Right breast upper-inner quadrant invasive lobular cancer; primary malignancy area of interest (blue arrow). S: Standard image, L: late image.

VOI: The Gray-Level Cooccurrence Matrix (GLCM), the Gray-Level Run Length Matrix (GLRLM), the Neighborhood Gray-Level Dependence Matrix (NGLDM), and the Gray-Level Zone, Length Matrix (GLZLM). GLCM represents that voxel pairs with specific SUV values can be found in a certain direction and at a certain distance. It is calculated using 1 voxel and 13 directions. GLRLM gives the size of homogeneous runs for each gray level using $13 \mathrm{di}$ rections, while NGLDM is calculated by calculating the gray level difference between a voxel and its 26 neighbors in three directions. GLZLM gives the size of homogeneous regions for each gray level in three dimensions. For the textural indices to be calculated software, there must be a VOI equal to or greater than 64 voxels that only contain one cluster, corresponding to a minimum volume of $2.12 \mathrm{ml}$ (voxel size of $4.07 \times 4.07 \times 2 \mathrm{~mm}$ ). Preferences for VOI with multiple clusters were manually selected by the operator. [20]

\section{Clinical and Histopathological Variables}

$\mathrm{T}$ and $\mathrm{N}$ stages, histological and nuclear grades, estrogen receptor (ER), progesterone receptor (PR), human epidermal growth factor receptor 2 (HER-2), and Ki-67 values of histopathological results are recorded of mastectomy (8 patients) or breast-conserving surgery (12 patients). Eight patients in the T1 stage and 12 patients in the T2 stage were included in the study. N1 and N2 patients are classified as $\mathrm{N}$ positive.

The objective study outcome was to evaluate the relationship between texture analysis findings of [18F] FDG PET/CT both standard and late images, T and $\mathrm{N}$ stages, and histopathological results (histologic and nuclear grades, presence of ER, PR and HER-2, and Ki67 level). In lobular breast cancer imaging, which has a relatively low level of FDG uptake, it is aimed to contribute to the diagnosis with the change of background activity in late images.

\section{Statistical Analysis}

The data were evaluated with the SPSS 25.0 (IBM, NY, USA) program. Comparison of standard and late images was evaluated with the Wilcoxon test, and the characteristics of tumor heterogeneity were evaluated with the Mann-Whitney U-test. Significant $p<0.05$ was considered. 


\section{Results}

Table 1 presents the demographic and histopathological characteristics of 20 ILC patients that we included in the study. Twelve breast-conserving surgery $(60 \%)$, eight mastectomies (40\%) were performed in median 32 days (1-56 days). Eight of the cases were pT1, 12 were pT2. The $\mathrm{N}$ stage of 8 ILC patients was $\mathrm{N}(-)$, the others were $\mathrm{N}(+)$.

The correlations between the textural analysis findings with standard and late imaging results of primary tumor are shown in Table 2.

SUV mean, max, Q1, Q2, Q3, peak $0.5 \mathrm{ml}$ and peak $1 \mathrm{ml}$, histo-skewness, kurtosis, excess kurtosis, entropy $\log 10$, entropy $\log 2$, GLCM homogeneity, energy, contrast, and dissimilarity, GLRLM LRE, SRLG and LRHGE, NGLDM contrast and busyness, GLZLM HGZE, SZHGE, ZLNU, and ZP values of late imaging were statistically higher than standard imaging $(\mathrm{p}<0.05)$.

When histopathological features were examined, significantly higher values were found in both standard and late images, especially in the ER-negative group (Tables 3, 4).

\section{Discussion}

ILC has the lowest sensitivity and specificity in diagnosis with conventional imaging methods due to growth pattern in invasive breast cancers.[21] According to the diagnosis of primary malignancy, lymph node and hematogenous metastasis diagnosis sensitivity and specificity are better, but the rates are still around $80 \%$. Due to the primary local diagnosis difficulties in ILC, it is likely that the case will be in the metastatic process at the time of diagnosis. In addition, compared to IDC, the probability of metastasis is higher, and the possibility of unexpected metastasis such as GIS, peritoneum, skin, and gynecological organs is also high.[22]

Therefore, in this study, we assumed that in addition to the management of malignancy in patients with ILC who have not previously been studied, the combination of [18F] FDG PET/CT late imaging and texture analysis may contribute to the diagnosis and/or better correlate with histopathological information. Actually, [18F] FDG PET/CT is not a routine examination for diagnosis in ILC because of the low [18F] FDG uptake due to low tumor cell density, diffuse infiltration in surrounding tissue, low Glut-1 expression, and low proliferation rate. Many different metabolic parameters such as SUV, MTV, TLG, and late imaging information have been used to increase sensitivity and specificity in breast tu-

\begin{tabular}{|c|c|c|c|c|}
\hline Characteristics & Value & $\mathbf{n}$ & $\%$ & Mean \pm SD \\
\hline Age & & & & $61.35 \pm 12.45$ \\
\hline \multirow[t]{2}{*}{ pT } & 1 & 8 & 40 & \\
\hline & 2 & 12 & 60 & \\
\hline \multirow[t]{2}{*}{$\mathrm{pN}$} & 0 & 8 & 40 & \\
\hline & 1 & 12 & 60 & \\
\hline \multirow[t]{3}{*}{ Nuclear grade } & 1 & 1 & 5 & \\
\hline & 2 & 10 & 50 & \\
\hline & 3 & 9 & 45 & \\
\hline \multirow[t]{3}{*}{ Histologic grade } & 1 & 2 & 10 & \\
\hline & 2 & 12 & 60 & \\
\hline & 3 & 6 & 30 & \\
\hline \multirow[t]{2}{*}{ HER-2 } & Negative & 15 & 75 & \\
\hline & Positive & 5 & 25 & \\
\hline Ki67<14 & Low & 7 & 35 & \\
\hline$\geq 14$ & High & 13 & 65 & \\
\hline \multirow[t]{2}{*}{ ER } & Negative & 3 & 15 & \\
\hline & Positive & 17 & 85 & \\
\hline \multirow[t]{2}{*}{ PR } & Negative & 7 & 35 & \\
\hline & Positive & 13 & 65 & \\
\hline
\end{tabular}

pT: Histopathologic tumor stage; pN: Histopathologic lymph node stage; HER-2: Human epidermal growth factor receptor 2; Ki-67: Cellular proliferation index; ER: Estrogen receptor; PR: Progesterone receptor

mors.[23] However, nearly all the studies constitute the more common IDC in the majority of cases. In seven studies investigating the contribution of late imaging with [18F] FDG PET/CT in invasive breast cancer, only $2 / 53,3 / 66,13 / 86,2 / 53,8 / 48$, and 2/38 ILC patients were included in the studies, respectively. $[17,18,24]$

In 86 invasive breast cancer cases, 13 of which were ILC with suspicion of local recurrence and distant metastasis, Garcia-Vicente et al.[25] shared that more malignancy could be detected by decreasing background activity in late images. In our study, especially the visualization of metastatic lymph node was seen in the late image of an ILC patient (Fig. 2).

Tumor heterogeneity with aggressive malignancy has been shown in many tumors. The techniques are proposed to define tumor heterogeneity for differentiation between tumor types, tumor grading, response monitoring, and outcome prediction in imaging. However, measuring tumor heterogeneity is not simple, information obtained from biopsy tissues are invasive and do not represent the whole tumor. Since intratumoral heterogeneity can be formed at very small areas, many studies have been designed to understand the heterogeneity of tumor with texture analysis information obtained from imaging which is non-invasive. Analysis methods for defining tumor heterogeneity are divided into four cat- 
Table 2 Comparison level and median values of textural indices between standard and late imaging

\begin{tabular}{|c|c|c|c|}
\hline Textural indicis & Standard imaging & Late imaging & $\mathbf{p}$ \\
\hline \multicolumn{4}{|l|}{ First Order } \\
\hline CONVENTIONAL_SUVbwmin & $2.4173 \pm 1.008$ & $2.5376 \pm 1.4672$ & 0.481 \\
\hline CONVENTIONAL_SUVbwmean & $3.93 \pm 1.78$ & $4.18 \pm 2.78$ & 0.003 \\
\hline CONVENTIONAL_SUVbwstd & $0.65 \pm 0.49$ & $1.08 \pm 0.85$ & $<0.001$ \\
\hline CONVENTIONAL_SUVbwmax & $5.11 \pm 3.10$ & $7.17 \pm 5.12$ & $<0.001$ \\
\hline CONVENTIONAL_SUVbwQ1 & $2.79 \pm 1.37$ & $3.27 \pm 2.11$ & 0.021 \\
\hline CONVENTIONAL_SUVbwQ2 & $3.23 \pm 1.79$ & $4.05 \pm 2.80$ & 0.005 \\
\hline CONVENTIONAL_SUVbwQ3 & $3.78 \pm 2.14$ & $4.95 \pm 3.40$ & 0.002 \\
\hline CONVENTIONAL_SUVbwSkewness & $0.74 \pm 0.40$ & $0.54 \pm 0.38$ & 0.096 \\
\hline CONVENTIONAL_SUVbwKurtosis & $2.84 \pm 0.86$ & $2.64 \pm 0.69$ & 0.466 \\
\hline CONVENTIONAL_SUVbwExcessKurtosis & $-0.11 \pm 1.06$ & $-0.36 \pm 0.69$ & 0.403 \\
\hline CONVENTIONAL_SUVbwpeakSphere $0.5 \mathrm{~mL}$ & $0.46 \pm 0.001$ & $0.46 \pm 0.001$ & 0.010 \\
\hline CONVENTIONAL_SUVbwpeakSphere $0.5 \mathrm{~mL}$ & $4.40 \pm 2.79$ & $5.68 \pm 4.61$ & 0.240 \\
\hline CONVENTIONAL_SUVbwpeakSphere $1 \mathrm{~mL}$ & $0.95 \pm 0.01$ & $0.96 \pm 0.001$ & 0.010 \\
\hline CONVENTIONAL_SUVbwpeakSphere $1 \mathrm{~mL}$ & $2.71 \pm 3.40$ & $3.90 \pm 4.82$ & 0.010 \\
\hline CONVENTIONAL_SUVbw & $9642.54 \pm 12637.45$ & $8245.07 \pm 11690.24$ & 0.240 \\
\hline CONVENTIONAL_TLG (mL) & $28.37 \pm 41.09$ & $33.50 \pm 51.35$ & 0.150 \\
\hline DISCRETIZED_SUVbwmean & $2.54 \pm 1.47$ & $11.17 \pm 5.70$ & $<0.001$ \\
\hline DISCRETIZED_SUVbwstd & $2.11 \pm 1.55$ & $3.47 \pm 2.68$ & $<0.001$ \\
\hline DISCRETIZED_SUVbwmax & $16.85 \pm 10.01$ & $23.10 \pm 15.27$ & $<0.001$ \\
\hline DISCRETIZED_SUVbwQ1 & $9.43 \pm 4.46$ & $10.94 \pm 6.69$ & 0.022 \\
\hline DISCRETIZED_SUVbwQ2 & $10.85 \pm 5.62$ & $13.48 \pm 8.99$ & 0.006 \\
\hline DISCRETIZED_SUVbwQ3 & $12.55 \pm 6.80$ & $16.28 \pm 10.87$ & 0.002 \\
\hline DISCRETIZED_SUVbwSkewness & $0.67 \pm 0.43$ & $0.54 \pm 0.38$ & 0.282 \\
\hline DISCRETIZED_SUVbwKurtosis & $2.84 \pm 0.96$ & $2.62 \pm 0.69$ & 0.422 \\
\hline DISCRETIZED_SUVbwExcessKurtosis & $-0.16 \pm 0.96$ & $-0.38 \pm 0.69$ & 0.422 \\
\hline DISCRETIZED_SUVbwpeakSphere $0.5 \mathrm{~mL}$ & $0.46 \pm 0.01$ & $0.46 \pm 0.01$ & $<0.001$ \\
\hline DISCRETIZED_SUVbwpeakSphere $0.5 \mathrm{~mL}$ & $5.67 \pm 4.61$ & $14.59 \pm 8.92$ & 0.003 \\
\hline DISCRETIZED_SUVbwpeakSphere $1 \mathrm{~mL}$ & $0.55 \pm 0.01$ & $0.95 \pm 0.01$ & 0.003 \\
\hline DISCRETIZED_SUVbwpeakSphere $1 \mathrm{~mL}$ & $8.92 \pm 11.09$ & $3.90 \pm 6.82$ & 0.174 \\
\hline DISCRETIZED_TLG (mL) & $94.43 \pm 135.89$ & $110.25 \pm 168.35$ & 0.001 \\
\hline DISCRETIZED_HISTO_Skewness & $2.97 \pm 1.39$ & $3.92 \pm 1.60$ & 0.007 \\
\hline DISCRETIZED_HISTO_Kurtosis & $13.28 \pm 8.98$ & $20.57 \pm 14.32$ & 0.007 \\
\hline DISCRETIZED_HISTO_ExcessKurtosis & $10.28 \pm 8.98$ & $17.57 \pm 14.32$ & 0.001 \\
\hline DISCRETIZED_HISTO_Entropy_log10 & $0.78 \pm 0.29$ & $0.97 \pm 0.31$ & 0.001 \\
\hline DISCRETIZED_HISTO_Entropy_log2 & $2.58 \pm 0.97$ & $3.23 \pm 1.02$ & $<0.001$ \\
\hline DISCRETIZED_HISTO_Energy & $0.15 \pm 0.10$ & $0.23 \pm 0.15$ & 0.001 \\
\hline SHAPE_Volume (mL) & $7.23 \pm 9.48$ & $6.18 \pm 8.77$ & 0.240 \\
\hline SHAPE_Volume (vx) & $296.70 \pm 388.85$ & $253.70 \pm 359.71$ & 0.240 \\
\hline SHAPE_Sphericity & $0.65 \pm 0.34$ & $0.63 \pm 0.38$ & 0.840 \\
\hline SHAPE_Surface $\left(\mathrm{mm}^{2}\right)$ & $1927.21 \pm 2068.76$ & $1599.16 \pm 1827.17$ & 0.162 \\
\hline SHAPE_Compacity & $2.42 \pm 1.47$ & $2.32 \pm 1.59$ & 0.616 \\
\hline \multicolumn{4}{|l|}{ Second order } \\
\hline GLCM_Homogeneity & $0.31 \pm 0.22$ & $0.42 \pm 0.26$ & 0.031 \\
\hline GLCM_Energy & $0.02 \pm 0.03$ & $0.06 \pm 0.09$ & 0.019 \\
\hline GLCM_Contrast & $7.29 \pm 11.59$ & $20.22 \pm 35.05$ & 0.026 \\
\hline GLCM_Correlation & $0.29 \pm 0.21$ & $0.29 \pm 0.22$ & 0.885 \\
\hline GLCM_Entropy_log10 & $1.20 \pm 0.78$ & $1.40 \pm 0.93$ & 0.142 \\
\hline GLCM_Entropy_log2 & $4.00 \pm 2.61$ & $4.65 \pm 3.09$ & 0.142 \\
\hline GLCM_Dissimilarity & $1.54 \pm 1.47$ & $2.51 \pm 2.47$ & 0.002 \\
\hline GLRLM_SRE & $0.69 \pm 0.36$ & $0.69 \pm 0.41$ & 0.990 \\
\hline
\end{tabular}




\begin{tabular}{lccc} 
Table 2 Cont. & & & \\
Textural indicis & Standard imaging & Late imaging & p \\
\hline GLRLM_LRE & $1.09 \pm 0.76$ & $1.55 \pm 1.17$ & $\mathbf{0 . 0 2 2}$ \\
GLRLM_LGRE & $0.01 \pm 0.01$ & $0.01 \pm 0.01$ & 0.867 \\
GLRLM_HGRE & $145.98 \pm 193.47$ & $262.96 \pm 428.28$ & 0.043 \\
GLRLM_SRLGE & $0.01 \pm 0.01$ & $0.01 \pm 0.16$ & 0.975 \\
GLRLM_SRHGE & $132.99 \pm 184.65$ & $249.44 \pm 413.91$ & $\mathbf{0 . 0 3 9}$ \\
GLRLM_LRLGE & $0.03 \pm 0.04$ & $0.02 \pm 0.05$ & 0.500 \\
GLRLM_LRHGE & $218.02 \pm 236.19$ & $325.00 \pm 490.76$ & 0.098 \\
GLRLM_GLNU & $18.61 \pm 24.02$ & $39.44 \pm 31.04$ & $\mathbf{0 . 0 2 1}$ \\
GLRLM_RLNU & $168.70 \pm 249.40$ & $184.62 \pm 28.11$ & 0.291 \\
GLRLM_RP & $0.66 \pm 0.35$ & $0.67 \pm 0.40$ & 0.889 \\
NGLDM_Coarseness & $0.03 \pm 0.02$ & $0.03 \pm 0.02$ & 0.860 \\
NGLDM_Contrast & $0.11 \pm 0.12$ & $0.19 \pm 0.23$ & $\mathbf{0 . 0 2 1}$ \\
NGLDM_Busyness & $0.32 \pm 0.36$ & $0.90 \pm 1.24$ & $\mathbf{0 . 0 1 7}$ \\
GLZLM_SZE & $0.30 \pm 0.23$ & $0.39 \pm 0.28$ & 0.084 \\
GLZLM_LZE & $3986.41 \pm 15142.89$ & $124.29 \pm 295.64$ & 0.260 \\
GLZLM_LGZE & $0.01 \pm 0.01$ & $0.01 \pm 0.03$ & 0.830 \\
GLZLM_HGZE & $142.56 \pm 180.46$ & $248.97 \pm 396.62$ & $\mathbf{0 . 0 4 6}$ \\
GLZLM_SZLGE & $69.02 \pm 114.44$ & $160.23 \pm 293.97$ & 0.254 \\
GLZLM_SZHGE & $0.003 \pm 0.003$ & $0.03 \pm 0.04$ & $\mathbf{0 . 0 3 7}$ \\
GLZLM_LZLGE & $87.53 \pm 331.98$ & $5.53 \pm 20.49$ & 0.254 \\
GLZLM_LZHGE & $210327.84 \pm 717060.47$ & $9897.67 \pm 17814.42$ & 0.223 \\
GLZLM_GLNU & $4.21 \pm 4.05$ & $5.16 \pm 5.52$ & 0.258 \\
GLZLM_ZLNU & $11.50 \pm 20.43$ & $29.09 \pm 47.34$ & $\mathbf{0 . 0 1 8}$ \\
GLZLM_ZP & $0.14 \pm 0.15$ & $0.24 \pm 0.21$ & $\mathbf{0 . 0 0 1}$ \\
\hline
\end{tabular}

GLCM: Gray-level cooccurrence matrix; GLRLM: Gray-level run length matrix; NGLDM: Neighborhood gray-level dependence matrix; GLZLM: Gray-level zone, length matrix. Bold values: $p<0.05$

egories as a category consisting of non-spatial methods, local spatial distribution methods, fractal analysis, and filters. Transformations in PET, magnetic resonance imaging, computed tomography, single photon emission computed tomography, and ultrasonography. However, due to limited software, lack of validation, and standardization, it cannot be assessed in routine practice.[19]

Tumor heterogeneity is also taking into account of [18F] FDG uptake.[26] The calculation of spatial heterogeneity with histogram-based features relied on the global computation of tumor heterogeneity only of the SUV values and not the spatial relations between voxels within the tumor.

Lee et al.[24] reported that late imaging showed a more significant correlation with prognostic factors in 38 invasive breast cancer cases. In addition, it was reported that a similar correlation was observed even in cases where the late image was taken earlier (100 min). In our study, we found higher number of correlated texture indicis in late imaging.

Dual time point application in [18F] FDG PET/ CT has been previously studied to evaluate variation of SUV-based parameters.[23] We found that 36 of 64 textural indices measuring tumor heterogeneity showed significant increases in late imaging compared to standard PET/CT acquisition. Tumor heterogeneity changes have been reported in $40.8 \%$ of tumors in delayed imaging using the gradient segmentation method using dual time point [18F] FDG PET/CT in patients with pancreatic adenocarcinoma.[27]

Larger tumors may have larger connected regions and provide larger values of the LRM-based variables. [28] In our study, statistically significant correlations found 15 in the standard image and 36 in the late image, and it was noteworthy that LRM-based texture indices have a significant correlation only in late images.

ER status is the determinant of the hormone therapy alternative and provides the most important prognostic information in breast cancer.[29] In our study, eight texture indicis correlations in standard image and 24 in late images were resulted of ER status.

The correlation between conventional quantitative FDG PET parameters and prognostic histopathological information in breast cancer has been described. 
Table 3 Significantly correlated texture indicis between standard imaging and histopathological features

\begin{tabular}{|c|c|c|c|c|}
\hline рт & $\mathbf{n}$ & Mean & Standart deviation & $\mathbf{p}$ \\
\hline \multicolumn{5}{|c|}{ SUVbwpeakSphere $0.5 \mathrm{~mL}$} \\
\hline 1 & 8 & 0.4631 & 0.00000 & $<0.001$ \\
\hline 2 & 12 & 0.9831 & 0.00000 & \\
\hline pN & $\mathbf{n}$ & Mean & Standart deviation & $\mathbf{p}$ \\
\hline \multicolumn{5}{|c|}{ SUVbwpeakSphere $0.5 \mathrm{~mL}$} \\
\hline 0 & 8 & 0.3831 & 0.00000 & 0.000 \\
\hline 1 & 12 & 0.9631 & 0.00000 & \\
\hline HER-2 & $n$ & Mean & Standart deviation & $\mathbf{P}$ \\
\hline \multicolumn{5}{|c|}{ SUVbwpeakSphere $0.5 \mathrm{~mL}$} \\
\hline 0 & 15 & 3.9591 & 2.73510 & 0.229 \\
\hline 1 & 5 & 5.7262 & 2.79975 & \\
\hline \multicolumn{5}{|c|}{ SUVbwpeakSphere $1 \mathrm{~mL}$} \\
\hline 0 & 15 & 0.9506 & 0.00000 & 0.016 \\
\hline 1 & 5 & 1.5606 & 0.00000 & \\
\hline Ki-67 & $\mathbf{n}$ & Mean & Standart deviation & $\mathbf{P}$ \\
\hline \multicolumn{5}{|c|}{ SUVbwpeakSphere $0.5 \mathrm{~mL}$} \\
\hline 0 & 7 & 0.4631 & 0.00000 & 0.000 \\
\hline 1 & 13 & 0.9831 & 0.00000 & \\
\hline ER & $\mathbf{n}$ & Mean & Std. deviation & $\mathbf{P}$ \\
\hline \multicolumn{5}{|c|}{ SUVbwpeakSphere $0.5 \mathrm{~mL}$} \\
\hline 0 & 3 & 0.0131 & 0.00000 & 0.002 \\
\hline 1 & 17 & 0.4631 & 0.00000 & \\
\hline \multicolumn{5}{|c|}{ SUVbwpeakSphere $1 \mathrm{~mL}$} \\
\hline 0 & 3 & 0.3206 & 0.00000 & 0.048 \\
\hline 1 & 17 & 0.9506 & 0.00000 & \\
\hline \multicolumn{5}{|c|}{ DISCRETIZED_SUVbwKurtosis } \\
\hline 0 & 3 & 2.3433 & 0.11211 & 0.034 \\
\hline 1 & 17 & 2.9281 & 1.01678 & \\
\hline \multicolumn{5}{|c|}{ DISCRETIZED_SUVbwExcessKurtosis } \\
\hline 0 & 3 & -0.6567 & 0.11211 & 0.034 \\
\hline 1 & 17 & -0.0719 & 1.01678 & \\
\hline \multicolumn{5}{|c|}{ GLRLM_LRHGE } \\
\hline 0 & 3 & 474.7468 & 409.44321 & 0.037 \\
\hline 1 & 17 & 172.7188 & 175.35681 & \\
\hline \multicolumn{5}{|c|}{ GLZLM_LZE } \\
\hline 0 & 3 & 22722.5233 & 39307.79580 & 0.015 \\
\hline 1 & 17 & 680.0421 & 1314.88247 & \\
\hline \multicolumn{5}{|c|}{ GLZLM_LZLGE } \\
\hline 0 & 3 & 496.4261 & 859.53050 & 0.016 \\
\hline 1 & 17 & 15.3822 & 40.55510 & \\
\hline \multicolumn{5}{|c|}{ GLZLM_LZHGE } \\
\hline 0 & 3 & 1072582.2201 & 1845429.95237 & 0.019 \\
\hline 1 & 17 & 58165.2966 & 144494.54711 & \\
\hline PR & $\mathbf{n}$ & Mean & Standart deviation & $\mathbf{P}$ \\
\hline \multicolumn{5}{|c|}{ SUVbwpeakSphere $0.5 \mathrm{~mL}$} \\
\hline 0 & 7 & 0.4631 & 0.00000 & 0.000 \\
\hline 1 & 13 & 0.4631 & 0.00000 & \\
\hline \multicolumn{5}{|c|}{ SHAPE_Sphericity } \\
\hline 0 & 7 & 0.8509 & 0.06183 & 0.049 \\
\hline 1 & 13 & 0.5377 & 0.38419 & \\
\hline
\end{tabular}

pT: Histopathologic tumor stage; pN: Histopathologic lymph node stage; HER-2: Human epidermal growth factor receptor 2; Ki-67: Cellular proliferation index; ER: Estrogen receptor; PR: Progesterone receptor; GLRLM: Gray-level run length matrix; GLZLM: Gray-level zone, length matrix 
Table 4 Significantly correlated texture indicis between late imaging and histopathological features

\begin{tabular}{|c|c|c|c|c|}
\hline pT & $n$ & Mean & Standart deviation & $\mathbf{P}$ \\
\hline \multicolumn{5}{|c|}{ SUVbwpeakSphere $0.5 \mathrm{~mL}$} \\
\hline 1 & 8 & 0.4627 & 0.00000 & 0.000 \\
\hline 2 & 12 & 0.8531 & 0.00000 & \\
\hline \multicolumn{5}{|c|}{ GLCM_Correlation } \\
\hline 1 & 8 & 0.1549 & 0.15626 & 0.019 \\
\hline 2 & 12 & 0.3729 & 0.22102 & \\
\hline \multicolumn{5}{|c|}{ GLRLM_GLNU } \\
\hline 1 & 8 & 9.2996 & 8.33259 & 0.040 \\
\hline 2 & 12 & 24.8181 & 29.11104 & \\
\hline \multicolumn{5}{|c|}{ GLZLM_LZE } \\
\hline 1 & 8 & 13.5034 & 11.71594 & 0.011 \\
\hline 2 & 12 & 198.1412 & 368.78322 & \\
\hline $\mathbf{p N}$ & $\mathbf{n}$ & Mean & Standart deviation & $\mathbf{P}$ \\
\hline \multicolumn{5}{|c|}{ SUVbwpeakSphere $0.5 \mathrm{~mL}$} \\
\hline 0 & 8 & 0.1651 & 0.00000 & 0.000 \\
\hline 1 & 12 & 0.7821 & 0.00000 & \\
\hline HER2 & $\mathbf{n}$ & Mean & Standart deviation & $\mathbf{P}$ \\
\hline \multicolumn{5}{|c|}{ SUVbwpeakSphere $0.5 \mathrm{~mL}$} \\
\hline 0 & 15 & 0.4631 & 0.00000 & 0.000 \\
\hline 1 & 5 & 1.0331 & 0.00000 & \\
\hline \multicolumn{5}{|c|}{ SUVbwpeakSphere $1 \mathrm{~mL}$} \\
\hline 0 & 15 & 0.3251 & 0.00000 & 0.009 \\
\hline 1 & 5 & 0.9506 & 0.00000 & \\
\hline Ki-67 & $\mathbf{n}$ & Mean & Standart deviation & $\mathbf{P}$ \\
\hline \multicolumn{5}{|c|}{ SUVbwpeakSphere $0.5 \mathrm{~mL}$} \\
\hline 0 & 7 & 0.4631 & 0.00000 & 0.000 \\
\hline 1 & 13 & 0.4631 & 0.00000 & \\
\hline \multicolumn{5}{|c|}{ DISCRETIZED_TLG (mL) (only For PET or NM) } \\
\hline 0 & 7 & 40.0124 & 40.90501 & 0.018 \\
\hline 1 & 13 & 148.0730 & 199.01920 & \\
\hline \multicolumn{5}{|c|}{ GLZLM_LZHGE } \\
\hline 0 & 7 & 2403.6852 & 2146.06460 & 0.074 \\
\hline 1 & 13 & 13932.8941 & 21207.86571 & \\
\hline \multicolumn{5}{|c|}{ GLZLM_ZLNU } \\
\hline 0 & 7 & 11.2081 & 12.65257 & 0.024 \\
\hline 1 & 13 & 38.7191 & 56.39899 & \\
\hline ER & $n$ & Mean & Standart deviation & $\mathbf{P}$ \\
\hline \multicolumn{5}{|c|}{ CONVENTIONAL_SUVbwstd } \\
\hline 0 & 3 & 1.8254 & 1.70764 & 0.049 \\
\hline 1 & 17 & 0.9446 & 0.60909 & \\
\hline \multicolumn{5}{|c|}{ CONVENTIONAL_SUVbwQ2 } \\
\hline 0 & 3 & 6.3688 & 5.95237 & 0.023 \\
\hline 1 & 17 & 3.6466 & 1.91821 & \\
\hline \multicolumn{5}{|c|}{ SUVbwpeakSphere $0.5 \mathrm{~mL}$} \\
\hline 0 & 3 & 0.1631 & 0.00000 & 0.002 \\
\hline 1 & 17 & 1.4631 & 0.00000 & \\
\hline \multicolumn{5}{|c|}{ SUVbwpeakSphere1mL } \\
\hline 0 & 3 & 0.8506 & 0.00000 & 0.036 \\
\hline 1 & 17 & 1.9506 & 0.00000 & \\
\hline \multicolumn{5}{|c|}{ DISCRETIZED_SUVbwstd } \\
\hline 0 & 3 & 5.8056 & 5.34400 & 0.010 \\
\hline 1 & 17 & 3.0574 & 1.93634 & \\
\hline \multicolumn{5}{|c|}{ DISCRETIZED_SUVbwQ2 } \\
\hline 0 & 3 & 20.8333 & 19.04162 & 0.027 \\
\hline 1 & 17 & 12.1765 & 6.21727 & \\
\hline
\end{tabular}


Table 4 Cont.

\begin{tabular}{|c|c|c|c|c|}
\hline pT & $\mathbf{n}$ & Mean & Standart deviation & $\mathbf{P}$ \\
\hline \multicolumn{5}{|c|}{ DISCRETIZED_SUVbwQ3 } \\
\hline 0 & 3 & 25.6667 & 23.35237 & \multirow[t]{2}{*}{0.011} \\
\hline 1 & 17 & 14.6176 & 7.26029 & \\
\hline \multicolumn{5}{|c|}{ DISCRETIZED_SUVbwSkewness } \\
\hline 0 & 3 & 0.2340 & 0.42251 & \multirow[t]{2}{*}{0.035} \\
\hline 1 & 17 & 0.5904 & 0.35583 & \\
\hline \multicolumn{5}{|c|}{ SUVbwpeakSphere $0.5 \mathrm{~mL}$} \\
\hline 0 & 3 & 0.4631 & 0.00000 & \multirow[t]{2}{*}{0.002} \\
\hline 1 & 17 & 0.4631 & 0.00000 & \\
\hline \multicolumn{5}{|c|}{ SUVbwpeakSphere $1 \mathrm{~mL}$} \\
\hline 0 & 3 & 0.9506 & 0.00000 & \multirow[t]{2}{*}{0.036} \\
\hline 1 & 17 & 0.9506 & 0.00000 & \\
\hline \multicolumn{5}{|c|}{ GLCM_Contrast (=Variance) } \\
\hline 0 & 3 & 59.9263 & 80.90714 & \multirow[t]{2}{*}{0.029} \\
\hline 1 & 17 & 13.2079 & 17.11548 & \\
\hline \multicolumn{5}{|c|}{ GLCM_Correlation } \\
\hline 0 & 3 & 0.4764 & 0.13178 & \multirow[t]{2}{*}{0.011} \\
\hline 1 & 17 & 0.2520 & 0.21984 & \\
\hline \multicolumn{5}{|c|}{ GLCM Dissimilarity } \\
\hline 0 & 3 & 4.7072 & 4.50903 & \multirow[t]{2}{*}{0.094} \\
\hline 1 & 17 & 2.1179 & 1.90183 & \\
\hline \multicolumn{5}{|c|}{ GLRLM_LRE } \\
\hline 0 & 3 & 1.7436 & 1.00954 & \multirow[t]{2}{*}{0.044} \\
\hline 1 & 17 & 0.9690 & 0.67850 & \\
\hline \multicolumn{5}{|c|}{ GLRLM_LGRE } \\
\hline 0 & 3 & 0.0333 & 0.05378 & 0.044 \\
\hline 1 & 17 & 0.0070 & 0.00770 & \\
\hline GLRLN & & & & \\
\hline 0 & 3 & 733.6547 & 967.85206 & 0.035 \\
\hline 1 & 17 & 179.9063 & 227.71957 & \\
\hline GLRLN & & & & \\
\hline 0 & 3 & 706.7432 & 939.95658 & 0.034 \\
\hline 1 & 17 & 168.7426 & 216.50435 & \\
\hline GLRLN & & & & \\
\hline 0 & 3 & 0.0792 & 0.13273 & 0.028 \\
\hline 1 & 17 & 0.0102 & 0.01333 & \\
\hline GLRLN & & & & \\
\hline 0 & 3 & 853.3071 & 1081.08451 & 0.039 \\
\hline 1 & 17 & 231.7710 & 279.89844 & \\
\hline NGLDI & & & & \\
\hline 0 & 3 & 0.4358 & 0.46514 & 0.042 \\
\hline 1 & 17 & 0.1505 & 0.14798 & \\
\hline GLZLN & & & & \\
\hline 0 & 3 & 0.0428 & 0.06999 & 0.035 \\
\hline 1 & 17 & 0.0075 & 0.00849 & \\
\hline GLZLN & & & & \\
\hline 0 & 3 & 684.9417 & 897.27092 & 0.035 \\
\hline 1 & 17 & 172.0280 & 210.33190 & \\
\hline GLZLN & & & & \\
\hline 0 & 3 & 486.2718 & 684.51046 & 0.033 \\
\hline 1 & 17 & 102.6936 & 143.53441 & \\
\hline GLZLN & & & & \\
\hline 0 & 3 & 30.5508 & 52.86789 & 0.017 \\
\hline 1 & 17 & 1.1165 & 3.31709 & \\
\hline PR & $\mathbf{n}$ & Mean & Standart deviation & $\mathbf{P}$ \\
\hline SUVbv & & & & \\
\hline 0 & 7 & 0.3231 & 0.00000 & 0.000 \\
\hline 1 & 13 & 0.7518 & 0.00000 & \\
\hline
\end{tabular}

pT: Histopathologic tumor stage; pN: Histopathologic lymph node stage; HER-2: Human epidermal growth factor receptor 2; Ki-67: Cellular proliferation index; ER: Estrogen receptor; PR: Progesterone receptor; GLCM: Gray-level cooccurrence matrix; GLRLM: Gray-level run length matrix; GLZLM: Gray-level zone, length matrix; NGLDM: Neighborhood gray-level dependence matrix 


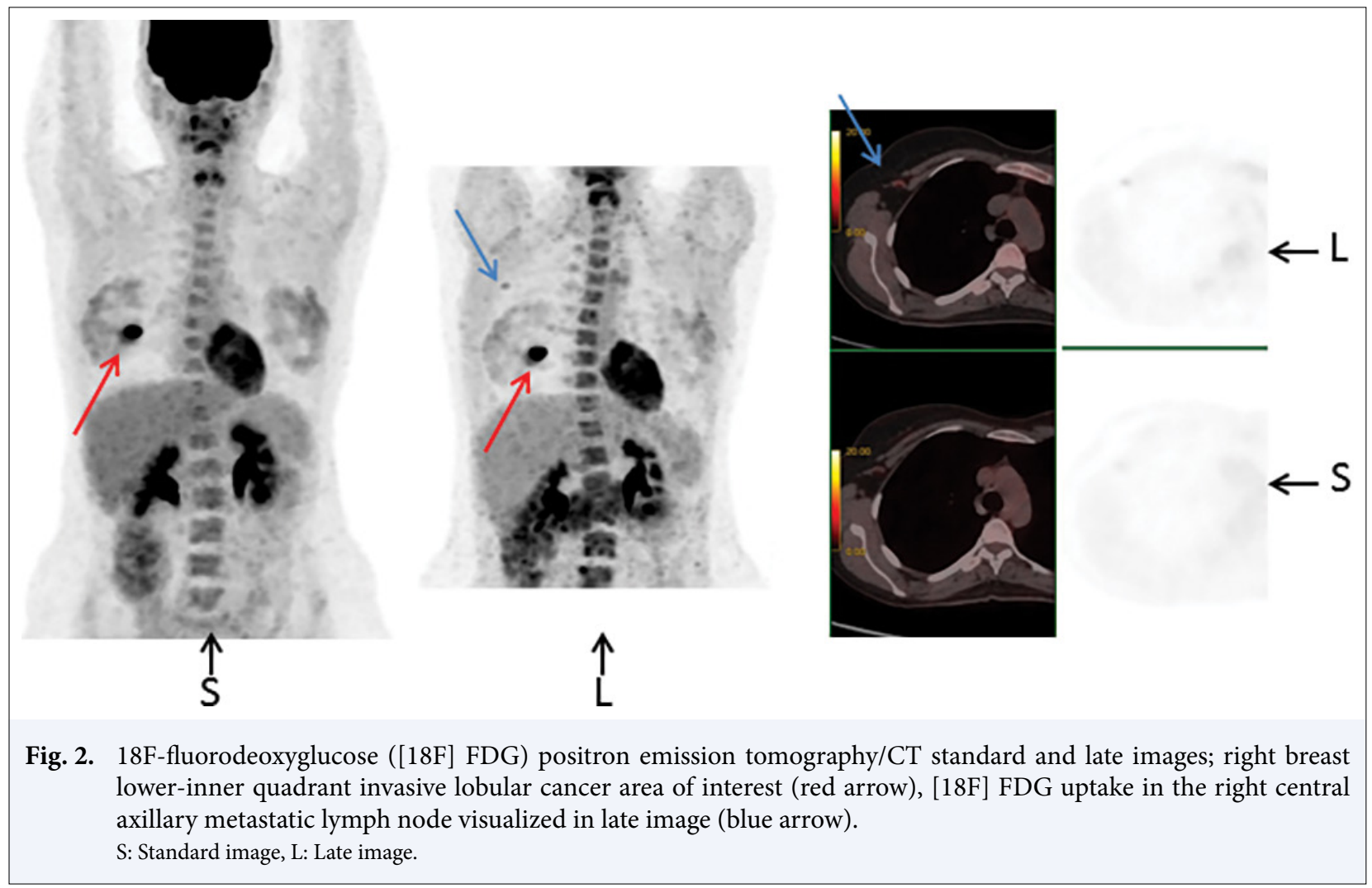

It is remarkable that in our study, especially GLRM and GLZLM-based texture indices also had this correlation. The studies can be performed with a high number of cases, early screening of cancer metastasis will be possible.

To the best of our knowledge, there are no studies evaluating the heterogeneity in ILC compared with histopathological variables in double time point PET/ CT. In addition, limited patient numbers and inconsistencies in the literature suggest that further analysis of heterogeneity in ILC is required. The major limitation of the study is the low number of patients and retrospective analysis. In our study, we aimed to investigate the correlation with prognostic histopathological information according to FDG affinity of ILC in standard and late images. Sensitivity and specificity values could not be given because there is no accepted threshold level for FDG uptake in breast lesions and it is not possible to perform a ROC analysis in which we can reach significant sensitivity and specificity levels with our existing patient number. ILC is not a common breast cancer, and FDG PET is not used much in staging. The lack of validation of the textural analysis has not been concluded and PET/CT spatial resolution cannot provide a clear conclusion about the small volume of tumors and questions of interpretation.

\section{Conclusion}

ILC shows relatively low uptake in [18F] FDG PET/ CT compared to IDC due to tumor characteristics. In our study, GLRM-based, GLZLM-based, and conventional/discretized indices have more correlation than other texture values. In addition, in late image, especially in the ER status, these correlations occur in much higher ( 15 vs. 36 ) in late image. For this method to be put into clinical practice, prospective and case-controlled studies are needed.

Peer-review: Externally peer-reviewed.

Conflict of Interest: All authors declared no conflict of interest.

Ethics Committee Approval: The study was approved by the Koc University Ethics Committee (No: 2019.359. IRB2.119, Date: 26/11/2019).

Financial Support: None declared.

Authorship contributions: Concept - F.O.F; Design F.O.F., H.S.; Supervision - F.O.F.; Funding - None; Materials - None; Data collection and/or processing - F.O.F.; Data analysis and/or interpretation - F.O.F., H.S.; Literature search - F.O.F., H.S.; Writing - F.O.F.; Critical review - F.O.F., H.S. 


\section{References}

1. The Lancet. GLOBOCAN 2018: Counting the toll of cancer. Lancet 2018;392(10152):985.

2. Narayan AK, Lehman CD. Mammography screening guideline controversies: Opportunities to improve patient engagement in screening. J Am Coll Radiol 2020;17(5):633-6.

3. Li CI, Uribe DJ, Daling JR. Clinical characteristics of different histologic types of breast cancer. $\mathrm{Br} \mathrm{J}$ Cancer 2005;93(9):1046-52.

4. Brawley O, Byers T, Chen A, Pignone M, Ransohoff D, Schenk M, et al. New American Cancer Society process for creating trustworthy cancer screening guidelines. JAMA 2011;306(22):2495-9.

5. Rakha EA, El-Sayed ME, Powe DG, Green AR, Habashy $\mathrm{H}$, Grainge MJ, et al. Invasive lobular carcinoma of the breast: Response to hormonal therapy and outcomes. Eur J Cancer 2008;44(1):73-83.

6. Li CI, Anderson BO, Daling JR, Moe RE. Trends in incidence rates of invasive lobular and ductal breast carcinoma. JAMA 2003;289(11):1421-4.

7. Scheidhauer K, Walter C, Seemann MD. FDG PET and other imaging modalities in the primary diagnosis of suspicious breast lesions. Eur J Nucl Med Mol Imaging 2004;31(Suppl 1):S70-9.

8. Nieweg OE, Kim EE, Wong WH, Broussard WF, Singletary SE, Hortobagyi GN, et al. Positron emission tomography with fluorine-18-deoxyglucose in the detection and staging of breast cancer. Cancer 1993;71(12):3920-5.

9. Buck AK, Schirrmeister H, Mattfeldt T, Reske SN. Biological characterisation of breast cancer by means of PET. Eur J Nucl Med Mol Imaging 2004;31(Suppl 1):S80-7.

10. Osborne JR, Port E, Gonen M, Doane A, Yeung H, Gerald W, et al. 18F-FDG PET of locally invasive breast cancer and association of estrogen receptor status with standardized uptake value: Microarray and immunohistochemical analysis. J Nucl Med 2010;51(4):543-50.

11. Hogan MP, Goldman DA, Dashevsky B, Riedl CC, Gönen M, Osborne JR, et al. Comparison of 18F-FDG PET/CT for systemic staging of newly diagnosed invasive lobular carcinoma versus invasive ductal carcinoma. J Nucl Med 2015;56(11):1674-80.

12. Dong A, Wang Y, Lu J, Zuo C. Spectrum of the breast lesions with increased 18F-FDG uptake on PET/CT. Clin Nucl Med 2016;41(7):543-57.

13. Bos R, van Der Hoeven JJ, van Der Wall E, van Der Groep P, van Diest PJ, Comans EF, et al. Biologic correlates of (18)fluorodeoxyglucose uptake in human breast cancer measured by positron emission tomography. J Clin Oncol 2002;20(2):379-87.

14. Jung NY, Kim SH, Kim SH, Seo YY, Oh JK, Choi HS, et al. Effectiveness of breast MRI and (18)F-FDG PET/ $\mathrm{CT}$ for the preoperative staging of invasive lobular carcinoma versus ductal carcinoma. J Breast Cancer 2015;18(1):63-72.

15. Choi WH, Yoo IR, O JH, Kim SH, Chung SK. The value of dual-time-point 18F-FDG PET/CT for identifying axillary lymph node metastasis in breast cancer patients. Br J Radiol 2011;84(1003):593-9.

16. Hahn S, Hecktor J, Grabellus F, Hartung V, Pöppel T, Kimmig R, et al. Diagnostic accuracy of dual-timepoint 18F-FDG PET/CT for the detection of axillary lymph node metastases in breast cancer patients. Acta Radiol 2012;53(5):518-23.

17. Ozen A, Altinay S, Ekmekcioglu O, Albayrak R, Muhammedoglu A, Yigitbas H, et al. Dual-time (18)FFDG PET/CT imaging in initial locoregional staging of breast carcinoma: Comparison with conventional imaging and pathological prognostic factors. Indian J Surg 2016;78(5):382-9.

18. Moon H, Noh WC, Kim HA, Kim EK, Park KW, Lee SS, et al. The relationship between estrogen receptor, progesterone receptor and human epidermal growth factor receptor 2 expression of breast cancer and the retention index in dual phase (18)F-FDG PET/CT. Nucl Med Mol Imaging 2016;50(3):246-54.

19. Alic L, Niessen WJ, Veenland JF. Quantification of heterogeneity as a biomarker in tumor imaging: A systematic review. PLoS One. 2014;9(10):e110300.

20. Nioche C, Orlhac F, Boughdad S, Reuzé S, Goya-Outi J, Robert C, et al. LIFEx: A freeware for radiomic feature calculation in multimodality imaging to accelerate advances in the characterization of tumor heterogeneity. Cancer Res 2018;78(16):4786-9.

21. Porter AJ, Evans EB, Foxcroft LM, Simpson PT, Lakhani SR. Mammographic and ultrasound features of invasive lobular carcinoma of the breast. J Med Imaging Radiat Oncol 2014;58(1):1-10.

22. Korhonen T, Kuukasjärvi T, Huhtala H, Alarmo EL, Holli K, Kallioniemi A, et al. The impact of lobular and ductal breast cancer histology on the metastatic behavior and long term survival of breast cancer patients. Breast 2013;22(6):1119-24.

23. Soussan M, Orlhac F, Boubaya M, Zelek L, Ziol M, Eder V, et al. Relationship between tumor heterogeneity measured on FDG-PET/CT and pathological prognostic factors in invasive breast cancer. PLoS One 2014;9(4):e94017.

24. Lee JY, Song HS, Choi JH, Hyun CL. Dual-time-point FDG uptake correlates with prognostic factors of invasive breast cancer: Clinical usefulness of early delayed scanning. Diagnostics (Basel) 2019;9(2):40.

25. Garcia-Vicente AM, Pérez-Beteta J, Pérez-García VM, Molina D, Jiménez-Londoño GA, Soriano-Castrejón A, et al. Metabolic tumor burden assessed by dual time 
point [(18)F]FDG PET/CT in locally advanced breast cancer: Relation with tumor biology. Mol Imaging Biol 2017;19(4):636-44.

26. Hatt M, Majdoub M, Vallières M, Tixier F, Le Rest CC, Groheux D, et al. 18F-FDG PET uptake characterization through texture analysis: Investigating the complementary nature of heterogeneity and functional tumor volume in a multi-cancer site patient cohort. J Nucl Med 2015;56(1):38-44.

27. Mena E, Sheikhbahaei S, Taghipour M, Jha AK, Vicente E, Xiao J, Subramaniam RM. 18F-FDG PET/ CT metabolic tumor volume and intratumoral heterogeneity in pancreatic adenocarcinomas: Impact of dual-time point and segmentation methods. Clin $\mathrm{Nucl}$ Med 2017;42(1):e16-21.

28. Orlhac F, Soussan M, Maisonobe JA, Garcia CA, Vanderlinden B, Buvat I. Tumor texture analysis in $18 \mathrm{~F}-$ FDG PET: Relationships between texture parameters, histogram indices, standardized uptake values, metabolic volumes, and total lesion glycolysis. J Nucl Med 2014;55(3):414-22.

29. Bentzon N, Büring M, Rasmussen BB, Mouridsen H, Kroman N. Prognostic effect of estrogen receptor status across age in primary breast cancer. Int J Cancer 2008;122(5):1089-94. 